\title{
Genome-wide identification and evolution of ATP-binding cassette transporters in the ciliate Tetrahymena thermophila: A case of functional divergence in a multigene family
}

\author{
Jie Xiong ${ }^{1,5}$, Lifang Feng ${ }^{1,4,5}$, Dongxia Yuan ${ }^{1}$, Chengjie Fu ${ }^{2,3^{*}}$, Wei Miao ${ }^{1 *}$
}

\begin{abstract}
Background: In eukaryotes, ABC transporters that utilize the energy of ATP hydrolysis to expel cellular substrates into the environment are responsible for most of the efflux from cells. Many members of the superfamily of $A B C$ transporters have been linked with resistance to multiple drugs or toxins. Owing to their medical and toxicological importance, members of the ABC superfamily have been studied in several model organisms and warrant examination in newly sequenced genomes.

Results: A total of $165 \mathrm{ABC}$ transporter genes, constituting a highly expanded superfamily relative to its size in other eukaryotes, were identified in the macronuclear genome of the ciliate Tetrahymena thermophila. Based on ortholog comparisons, phylogenetic topologies and intron characterizations, each highly expanded $A B C$ transporter family of T. thermophila was classified into several distinct groups, and hypotheses about their evolutionary relationships are presented. A comprehensive microarray analysis revealed divergent expression patterns among the members of the $A B C$ transporter superfamily during different states of physiology and development. Many of the relatively recently formed duplicate pairs within individual $A B C$ transporter families exhibit significantly different expression patterns. Further analysis showed that multiple mechanisms have led to functional divergence that is responsible for the preservation of duplicated genes.

Conclusion: Gene duplications have resulted in an extensive expansion of the superfamily of $A B C$ transporters in the Tetrahymena genome, making it the largest example of its kind reported in any organism to date. Multiple independent duplications and subsequent divergence contributed to the formation of different families of $A B C$ transporter genes. Many of the members within a gene family exhibit different expression patterns. The combination of gene duplication followed by both sequence divergence and acquisition of new patterns of expression likely plays a role in the adaptation of Tetrahymen a to its environment.
\end{abstract}

\section{Background}

The ATP-binding cassette $(\mathrm{ABC})$ transporter superfamily of genes is one of the largest in the genomes of both bacteria and eukaryotes [1]. Using hydrolysis of ATP to $\mathrm{ADP}$ to generate energy, $\mathrm{ABC}$ transporters move a wide variety of substrates across membranes, including ions,

\footnotetext{
* Correspondence: cjfu1981@gmail.com; miaowei530@yeah.net ${ }^{1}$ Key Laboratory of Aquatic Biodiversity and Conservation, Institute of Hydrobiology, Chinese Academy of Sciences, Wuhan, 430072, PR China ${ }^{2}$ State Key Laboratory of Freshwater Ecology and Biotechnology, Institute of Hydrobiology, Chinese Academy of Sciences, Wuhan, 430072, PR China Full list of author information is available at the end of the article
}

sugars, amino acids, polypeptides, toxic metabolites, xenobiotics, and drugs. Therefore, they provide nutrients to a cell as well as protect it from both internally produced and exogenous toxins [2,3]. Eukaryotic ABC transporters usually consist of two types of domains, a transmembrane domain (TMD) and a nucleotide-binding domain (NBD). Many ABC proteins include two of each type of domain and are so-called full transporters. Others are half transporters, containing one TMD and one NBD, and generate a functional unit by forming a homo- or heterodimer [2]. Based on their primary sequences and the organization of their domains, the 
human $A B C$ genes were classified into seven families, from $\mathrm{ABCA}$ to $\mathrm{ABCG}$ [4]; an eighth $\mathrm{ABCH}$ family was discovered in the analysis of the Drosophila melanogaster genome [5].

In humans, mutations of many $\mathrm{ABC}$ genes are linked to hereditary disorders, such as adrenoleukodystrophy and cystic fibrosis [5,6], and proteins coded for by many genes in the $A B C$ families $B, C$ and $G$ function as drug efflux transporters [7]. In parasites, products of $A B C$ genes have been implicated as factors contributing to resistance against chemotherapeutics [8], and in insects, $\mathrm{ABC}$ genes have been linked to pesticide resistance [9-11]. These medical and toxicological roles make ABC transporters important in pharmacological research [12], therapeutic applications [13], and toxicology [14]. Extensive investigations of $A B C$ transporters in bacteria and multicellular eukaryotes have been done [5,15-22], but studies in unicellular eukaryotes other than yeasts have been limited to parasitic species [23,24]. Clearly, the $A B C$ transporter superfamily in unicellular free-living species requires additional study.

Tetrahymena is a free-living ciliated protist found in freshwater environments around the world [25]. At the cellular level, its structural and functional complexity is equal to or greater than that of individual metazoan cells. Studies on Tetrahymena have led to numerous scientific breakthroughs, and a number of molecular genetic technologies and genomic resources have recently been developed [26-28]. In particular, the Tetrahymena Genome Database (TGD, http://www.ciliate. org) and Tetrahymena genome expression database (TGED, http://tged.ihb.ac.cn) $[28,29]$ provide the opportunity for analysis of both the functional and evolutionary characteristics of gene families at the genomic level in this model organism [30].

In the present study, we identified $165 \mathrm{ABC}$ transporter genes in the Tetrahymena thermophila macronuclear genome. The locations of introns, evolutionary relationships and expression patterns of these genes were characterized. A detailed analysis showed that the evolutionary and functional divergence of Tetrahymena $\mathrm{ABC}$ transporters has resulted from a combination of gene duplication, mutation, pseudogenization, and changes in gene expression.

\section{Results and Discussion}

Identification and classification of $A B C$ transporter genes in $T$. thermophila

A total of 165 putative $\mathrm{ABC}$ transporter genes were identified in T. thermophila (Additional file 1), making it the largest superfamily of $\mathrm{ABC}$ transporter genes described in any species to date. All of the genes could be grouped into eight families based on organization of domains, BlastP scores, and placement in phylogenetic trees. Their phylogenetic relationships are shown in the ML tree (Figure 1).

The ABCA family comprises 29 full transporters and 4 half transporters. The gene model for one full $A B C$ transporter gene, $A B C A 12$ (TGD accession NO: TTHERM_00694300), was predicted incorrectly by TIGR. Our RT-PCR results showed that this "monster" gene was, in fact, two full-transporter genes, i.e., $A B C A 12-1$ and $A B C A 12-2$ (data not shown). In vertebrates, the ABCA family is composed entirely of full transporters, and in the plant Arabidopsis all are half transporters [31]. However, both full and half transporters have been found in another protist, Dictyostelium [32]. No $A B C A$ genes have been identified in yeast [33] (Table 1). Three ABCA genes (ABCA1, $A B C A 8$, and $A B C A 15)$ in Tetrahymena are highly similar to the suABCA gene in sea urchins, which was found in sperm membrane vesicles and is involved in changing the immature sperm membrane to produce the terminally differentiated mature spermatozoa [34]. These three Tetrahymena ABCA genes were markedly up-regulated during starvation, and the high levels of expression were maintained during conjugation. Starvation induces physiological changes that prepare cells of different matingtypes to form mating pairs [35]; therefore, these ABCA genes may be involved in inducing and/or sustaining conjugation.

The ABCB family consists of 13 full transporters and 13 half transporters. Among those, one full transporter, $A B C B 23$ (TGD accession NO: TTHERM_00803570), possesses both one ABCB-like NBD (E-value: $6 \mathrm{e}^{-61}$ ) and one ABCC-like NBD (E-value: $3 \mathrm{e}^{-50}$ ). The mixed structure of this gene was verified by sequencing its cDNA between the first NBD and second TMD (Additional file 2). The $A B C B 23$ gene was grouped into the $A B C B$ family according to the higher similarity of its NBD (lower E-value) to ABCB-like NBD.

Two ABCB genes $(A B C B 12$ and $A B C B 13)$ in Tetrahymena were predicted to have a mitochondrial localization by the Predotar program (http://urgi.versailles.inra. $\mathrm{fr} /$ predotar/predotar.html). The half transporter ABCB12 has an ABC_ATM1 domain (cd03253, E-value: $2 \mathrm{e}^{-65}$ ) that resembles the NBD of human ABCB7 and the yeast ATM1 gene, both of which are involved in the transport of the $\mathrm{Fe} / \mathrm{S}$ binding protein into mitochondria [36]. There is an ABC_MTABC3 domain (cd03249, E-value: $1 \mathrm{e}^{-63}$ ) in $\mathrm{ABCB} 13$ that resembles the NBD of human ABCB6, which has been shown to be associated with iron transport and is located in the mitochondrial membrane [37].

The human $A B C B 1$ gene MDR1 (multidrug resistance gene) encodes a well-studied p-glycoprotein that has been shown to confer resistance to or transport a wide variety of anticancer agents. The MDR protein is 


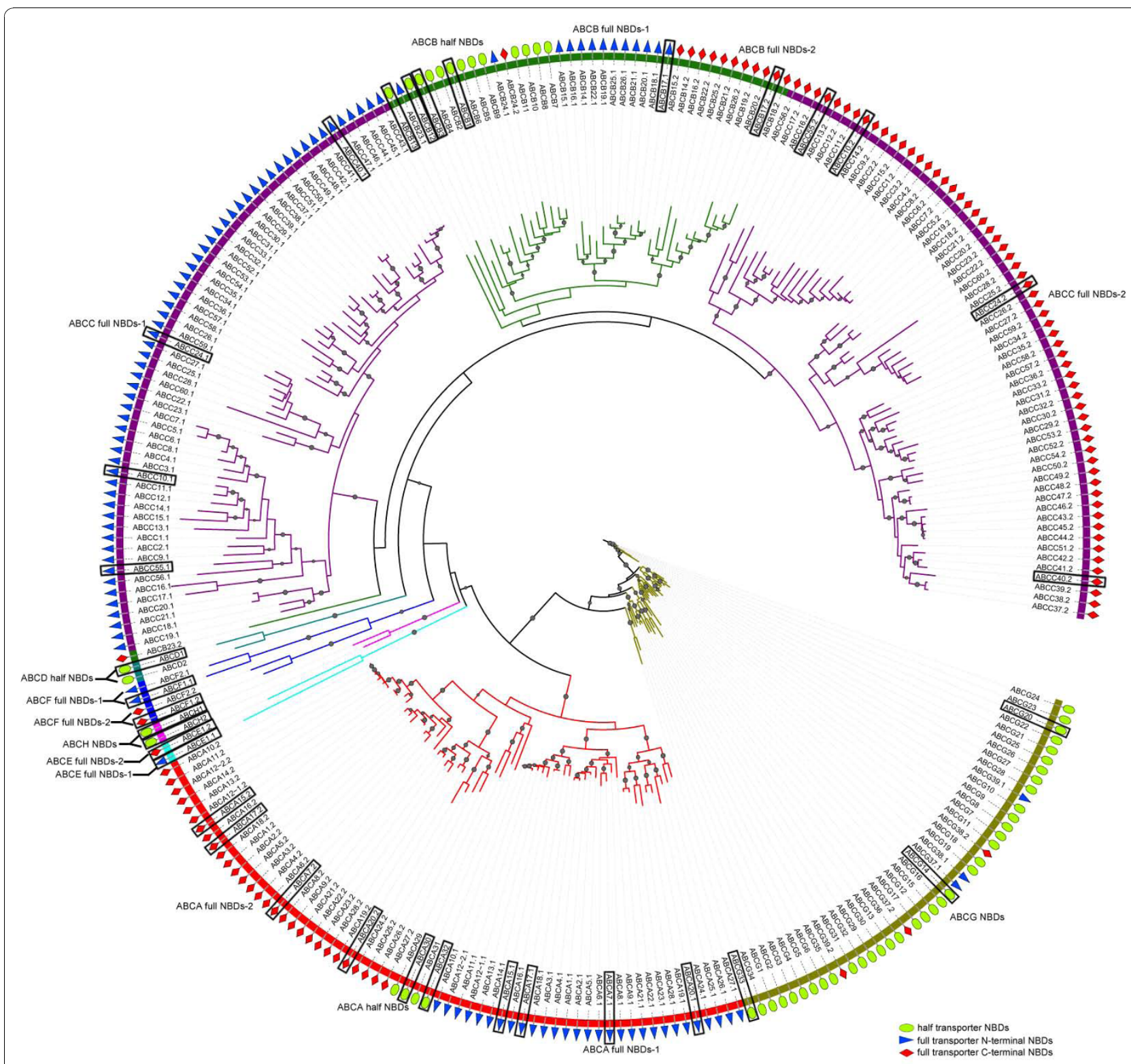

Figure 1 Unrooted ML tree of ABC transporter NBDs in $T$. thermophila. Each color represents a family of ABC transporters. Genes that have an ortholog in the genome of Paramecium are marked with black panes. Bootstrap values $>50 \%$ consensus are marked with gray circles, and their sizes indicate the levels of consensus.

thought to transport both endo- and xenobiotics and protect cells from toxic agents [38]. The $A B C B 15$ (TGD accession NO: TTHERM_00240450) of T. thermophila shares a $32.3 \%$ amino acid identity with MDR1, which is higher than that between the human p-glycoprotein and its ortholog STE6 in the yeast (26.2\%) [39]. A protein composed of both 66 and $96 \mathrm{kDa}$ subunits has been reported to function in $T$. pyriformis as an efflux pump similar to mammalian MDR1 $[40,41]$, and a putative p-glycoprotein in T. pyriformis (GenBank accession No. CAD55936) has been described. The $A B C B 15$ of
T. thermophila shares a $35.2 \%$ amino acid identity with a putative p-glycoprotein of T. pyriformis and is induced by DDT, but not by either TCDD or $\beta$-estradiol (Additional file 3). Thus, the $A B C B 15$ protein might function as a substrate-specific MDR,

The ABCC family comprises 60 full transporters and thus represents the largest family of $\mathrm{ABC}$ transporters in T. thermophila. In the human genome, nine $A B C C$ genes encode the multidrug resistance proteins (MRPs), which contain a core domain of TMD similar to pglycoprotein, with the difference that some of them 
Table 1 Comparison of ABC transporters in Tetrahymena thermophila and other eukaryotes

\begin{tabular}{|c|c|c|c|c|c|c|c|c|c|}
\hline Family & Structure & $\mathrm{Tt}$ & $\mathrm{Pt}$ & $\mathrm{Bb}$ & Py & $\mathrm{Dd}$ & At & Sc & $\mathrm{Hs}$ \\
\hline \multirow[t]{2}{*}{$A B C A$} & $(T M D-N B D)_{2}$ & $29^{a}$ & 20 & 0 & 0 & 7 & 0 & 0 & 13 \\
\hline & TMD-NBD & 4 & 4 & 0 & 0 & 5 & $17^{\mathrm{b}}$ & 0 & 0 \\
\hline \multirow[t]{2}{*}{$A B C B$} & $(\mathrm{TMD}-\mathrm{NBD})_{2}$ & 13 & 7 & 0 & 1 & 2 & 22 & 1 & 4 \\
\hline & TMD-NBD & 13 & 7 & 4 & 6 & 7 & 5 & 3 & 7 \\
\hline $\mathrm{ABCC}$ & $(\mathrm{TMD}-\mathrm{NBD})_{2}$ & 60 & $46^{c}$ & 4 & 1 & 14 & 15 & 7 & 12 \\
\hline \multirow[t]{2}{*}{$A B C D$} & $(\mathrm{TMD}-\mathrm{NBD})_{2}$ & 0 & 0 & 0 & 0 & 0 & 1 & 0 & 0 \\
\hline & TMD-NBD & 2 & 4 & 0 & 0 & 3 & 1 & 2 & 4 \\
\hline ABCE & NBD-NBD & 1 & 2 & 1 & 1 & 1 & 2 & 1 & 1 \\
\hline $\mathrm{ABCF}$ & NBD-NBD & 2 & 2 & 1 & 1 & 4 & 5 & 5 & 3 \\
\hline \multirow[t]{2}{*}{$\mathrm{ABCG}$} & $(\mathrm{TMD}-\mathrm{NBD})_{2}$ & 3 & 0 & 0 & 0 & 15 & 13 & 10 & 0 \\
\hline & TMD-NBD & 36 & 17 & 0 & 1 & 6 & 29 & $1^{d}$ & 5 \\
\hline Other $^{\mathrm{e}}$ & NBD & 2 & 3 & 0 & 3 & 4 & 15 & 2 & 2 \\
\hline Total & - & 165 & 112 & 10 & 14 & 68 & 125 & 32 & 50 \\
\hline Number of Genes & - & 24725 & 39642 & 3671 & 5878 & 12500 & 25498 & 5538 & 22287 \\
\hline Percent & - & $0.667 \%$ & $0.283 \%$ & $0.272 \%$ & $0.238 \%$ & $0.544 \%$ & $0.490 \%$ & $0.577 \%$ & $0.224 \%$ \\
\hline
\end{tabular}

$\mathrm{Tt}=$ Tetrahymena thermophila, $\mathrm{Pt}=$ Paramecium tetraurelia, $\mathrm{Bb}=$ Babesia bovis (has the minimum predicted gene number in sequenced apicomplexans), $\mathrm{Py}=$ Plasmodium yoelii yoelii (has the maximum predicted gene number in sequenced apicomplexans), Dd = Dictyostelium discoideum, At = Arabidopsis thaliana, Sc = Saccharomyces cerevisiae, $\mathrm{Hm}=$ Homo sapiens.

ane incorrectly predicted gene was identified.

${ }^{b_{i n c l u d e s}}$ a gene annotated as (TMD-NBD) ${ }_{2}$ in a previous report, but revised as TMD-NBD in the current version of the annotation.

cincludes 5 genes with a structure TMD-NBD and 2 genes with a structure NBD.

d this gene has three TMDs and one NBD.

includes the $\mathrm{ABCH}$ family of gene.

have an extra TMD at their N-terminal region to form the structure TMD-TMD-NBD-TMD-NBD. MRPs transport xenobiotics coupling glutathione (GSH), which is a major feature distinguishing them from p-gp; therefore MRPs also are recognized as GS-X pumps [38,42]. In T. thermophila, no extra TMD was found at the Nterminal region of $A B C C$ proteins, but an extra GST_Mu domain was present at the C-terminal region of $A B C C 52$ (Additional file 1). Xenobiotics are fused with GSH by glutathione $S$-transferases at phase II, and then the complexed substances are transported by MRPs at phase III, suggesting a coordinated action of GSTs and MRPs [43]. ABCC52 is a MRP-like gene and the GST_Mu domain may indicate that it functions similarly to recognize such GST-complexed substances.

The ABCD family contains only 2 half transporters. The ABCD families of most other organisms also consist of a low number of half transporters except for that of the plant Arabidopsis thaliana, which includes both a full and a half transporter [44] (Table 1). Members in this family that have been studied are all targeted to the peroxisome, where they regulate the transport of longchain fatty acids [45]. In yeast, two ABCD half transporters (Pxa1p and Pxa2p) were found, and these two half transporters form a heterodimer that functions in the $\beta$-oxidation of long-chain fatty acids [46]; In humans, there are four ABCD genes [47], the human ABCD1 is responsible for the X-linked form of adrenoleukodystrophy as a homodimer [48]. The $A B C D 1$ and $A B C D 2$ genes of T. thermophila exhibited the same pattern of expression (Pearson Correlation Coefficient, PCC = 0.948) during the physiological/developmental stages of growth, starvation and conjugation (Additional file 4), suggesting that a heterodimer may also be their functional form.

The ABCE and $A B C F$ families each contain only two NBDs without TMDs in T. thermophila. Almost all eukaryotes also have only one $A B C E$ gene except A. thaliana, which has two [31]. In Tetrahymena, also one $A B C E$ gene has been identified. In animals, ABCE has been shown to inhibit RNase L, a double-stranded RNA nuclease, and is referred to as RLi [49]. Many intron positions within $A B C E 1$ genes are conserved in animals and plants, especially in vertebrates (Additional file 5); therefore, it has been suggested that most of these introns (e.g., those in human, mouse and rat $\mathrm{ABCE} 1)$ were ancestral in nature and that many introns have been lost in other species (e.g., Ciona intestinalis, Anopheles gambiae, Drosophila melanogaster, Caenorhabditis elegans, Dictyostelium discoideum, Plasmodium falciparum) [15]. The ABCE1 of T. thermophila contains 6 introns; however, it shares no intron positions with ABCE1s of animals and only one with that of Arabidopsis, suggesting that the timing and mechanism of intron gain and loss in the $A B C E 1$ gene of T. thermophila may have differed from those of opisthokonts and plants. For the ABCF family, a well studied gene is the GCN20 of yeast, which has been shown to regulate translation in 
amino acid-starved cells by interaction with eukaryotic initiation factor 2 (eIF2) and ribosomes [50]. In Tetrahymena, two ABCF genes were found; one of the two genes (TGD accession NO: TTHERM_01014620) showed a high expression level in the three stages of T. thermophila life cycle, indicated a constitutive function of this gene.

The ABCG family includes 3 full transporters and 36 half transporters. Members of the ABCG family are structured as NBD-TMD or NBD-TMD-NBD-TMD that have a TMD at the C-terminal region of NBD, an orientation inverse to that of the $\mathrm{ABCA}, \mathrm{ABCB}, \mathrm{ABCC}$ and $A B C D$ families. Human $A B C G 2$, first identified in cells from placentas and breast cancers, is a multidrug resistance $A B C$ transporter gene different from both MDR1 or MRP1 and is known as breast cancer resistant protein (BCRP). A half transporter, BCRP functions as a homodimer or tetramer bridged with disulfide bonds [51,52]. Expression of $A B C G 25$ is induced by DDT T. thermophila, and it shares $31.8 \%$ amino acid identity with BCRP, suggesting that it might have the same basic function (Additional file 3).

The ABCH family was identified in Drosophila [47], and lacks members in mammals and C. elegans. The function of $\mathrm{ABCH}$ is unknown. Tetrahymena has 2 $\mathrm{ABCH}$ genes, both of which have a single NBD. The $A B C H 1$ gene of the social amoeba $D$. discoideum has the same structure [32]. However, genes of the $\mathrm{ABCH}$ family encode a half transporter with an NBD-TMD structure in D. melanogaster and A. gambiae [47].

\section{The evolution of $\mathrm{ABC}$ transporters in Tetrahymena}

All N-terminal NBDs (NBDs-1) and C-terminal NBDs (NBDs-2) of full transporters and NBDs of half transporters (NBDs-half) were used to construct the ML tree (Figure 1). It showed that NBDs in the same family clustered together except for the ABCC family (Figure 1). The relationship of the eight families was similar to that previously reported in Dictyostelium [32]. Comparative genomics analyses of $\mathrm{ABC}$ transporters suggested that the ancestral $\mathrm{ABC}$ transporters arose before the differentiation of prokaryotes and eukaryotes [53-55]. In another analysis using 19 evolutionarily diverse eukaryotes, those NBDs in the same family still cluster together (unpublished result). These results suggest that the eight $A B C$ transporter families may have diverged prior to the last universal common ancestor (LUCA) of existing eukaryotes.

Many proteins are composed of at least two domains, and it has become clear that the formation of new domain combinations is an important mechanism in protein evolution [56]. In most cases, but not always, the eukaryotic $\mathrm{ABC}$ transporters are composed of two or four domains through various combinations of TMDs and NBDs. Half transporters are composed of a single TMD domain fused to an NBD domain, a structural organization that could be symbolized as TMD-ABC or ABC-TMD (ABCG family) depending on the $\mathrm{N}$ - or C-terminal location of the TMD domain. Full-size transporters are probably generated by duplication and fusion of "half-size" transporters and are symbolized as [TMD$\mathrm{NBD}]_{2}$ and [NBD-TMD] $]_{2}$ (ABCG family) [55,57-59]. In our analysis, three phylogenetic relationships were found among the three kinds NBDs (NBDs- 1 and NBDs- 2 from full transporters and NBD-Half from half transporters) in an unrooted tree, corresponding to the $A B C A$, $\mathrm{ABCB}$ and $\mathrm{ACBG}$ families respectively. In the $\mathrm{ABCA}$ family, NBDs-2 (A-2) showed a distant relationship to NBDs-1 (A-1) and closer relationship to NBDs-Half (A-Half) (Figure 2), which indicated the possibility that the ABCA full transporter fused from two distinct half transporters, which we refer to as "heterogenous" fusion. In the $A B C B$ family, NBDs-2 (B-2) showed a close relationship to NBDs-1 (B-1) and a more distant relationship to NBDs-Half (B-Half) (Figure 3), which indicated the possibility that the $\mathrm{ABCB}$ full transporter formed by fusion of two high similarity half transporters, referred to as "homogenous" fusion. The ABCG family possesses three full transporters with a reverse domain organization. Neither NBDs-1 (G-1) nor NBDs-2 (G-2) associated into separate cluster but, instead, were dispersed among the cluster of NBDs-Half (G-Half) (Figure 4). Since there are no ABCG full genes in the P. tetraurelia genome, this result indicates that the $T$. thermophila ABCG full transporters originated after these two species diverged. NBDs-1 and NBDs-2 of two full genes (ABCG37 and ABCG38) are in the same group (group4) (Figure 4), while two NBDs of the other ABCG full transporter (ABCG39) cluster into two groups (group3 and group5). Although the ancestor could not be determined in an unrooted tree, these three pairs of NBDs seem to correspond to "homogenous" and "heterogenous" fusions. In addition, the ABCC family members were all full transporters with distant relationship of NBDs-1 and NBDs-2, also may have undergone a heterogenous fusion process.

Possession of a large number and variety of $A B C$ transporters is a significant characteristic of T. thermophila, and we carried out an analysis to discover whether this results from numerous, lineage-specific duplications and when these duplications occurred. First, a phylogeny of NBDs was used to investigate the evolution of all of the $165 \mathrm{~T}$. thermophila ABC transporter genes, using both full transporters (NBDs-1 and NBDs-2) and half transporters (NBDs-half) (Figure 1). A strong correlation was observed between the conservation of intron positions and phylogenetic relationships of $\mathrm{ABC}$ transporters, except for some scattered examples 


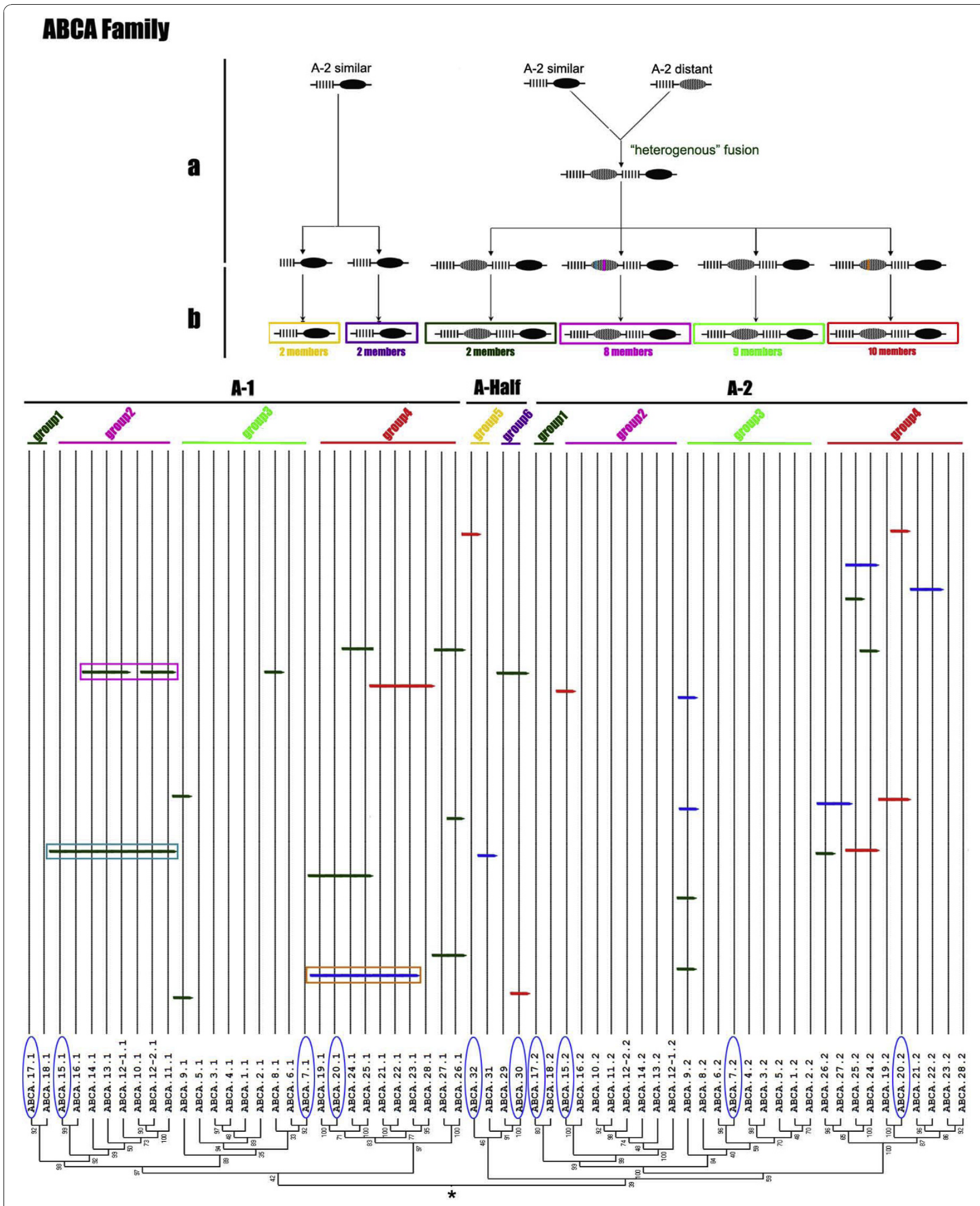

Figure 2 Phylogenetic tree and a proposed evolutionary scheme of the ABCA family. Above: the proposed events in the evolution of the ABCA family. a, putative full transporter formation process and divergence of the ABCA transporter with gain and loss of introns, the colored vertical bars in the NBD region represent conserved introns; $b$, divergence of the ABCA transporter gene to give rise to each group. Below: part of tree from Figure 1 with introns locations of NBDs, the asterisk represent the root in the midpoint. Each group was marked as a line with the same color to the pane in b; genes that have a Paramecium ortholog were marked with a blue ellipse. 


\section{ABEB family}
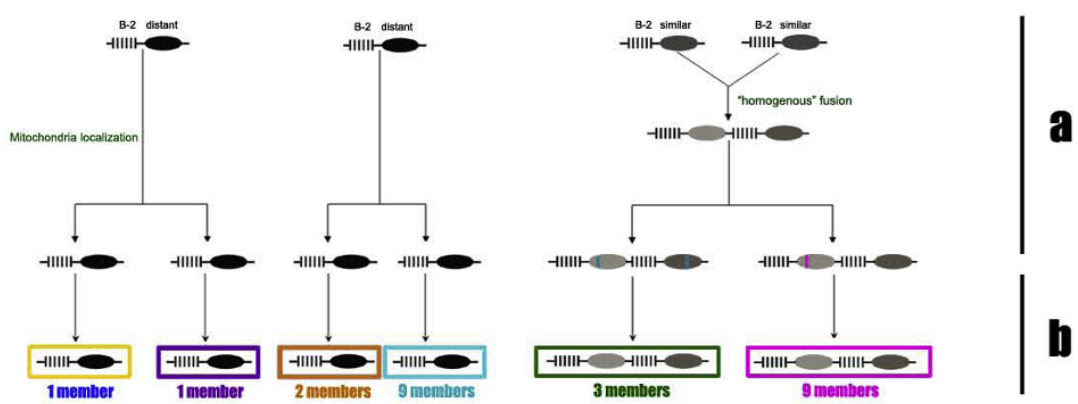

B-Halif

B-2
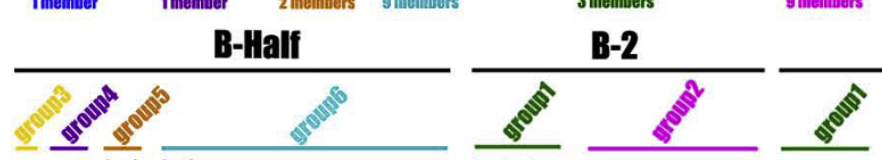

B-1
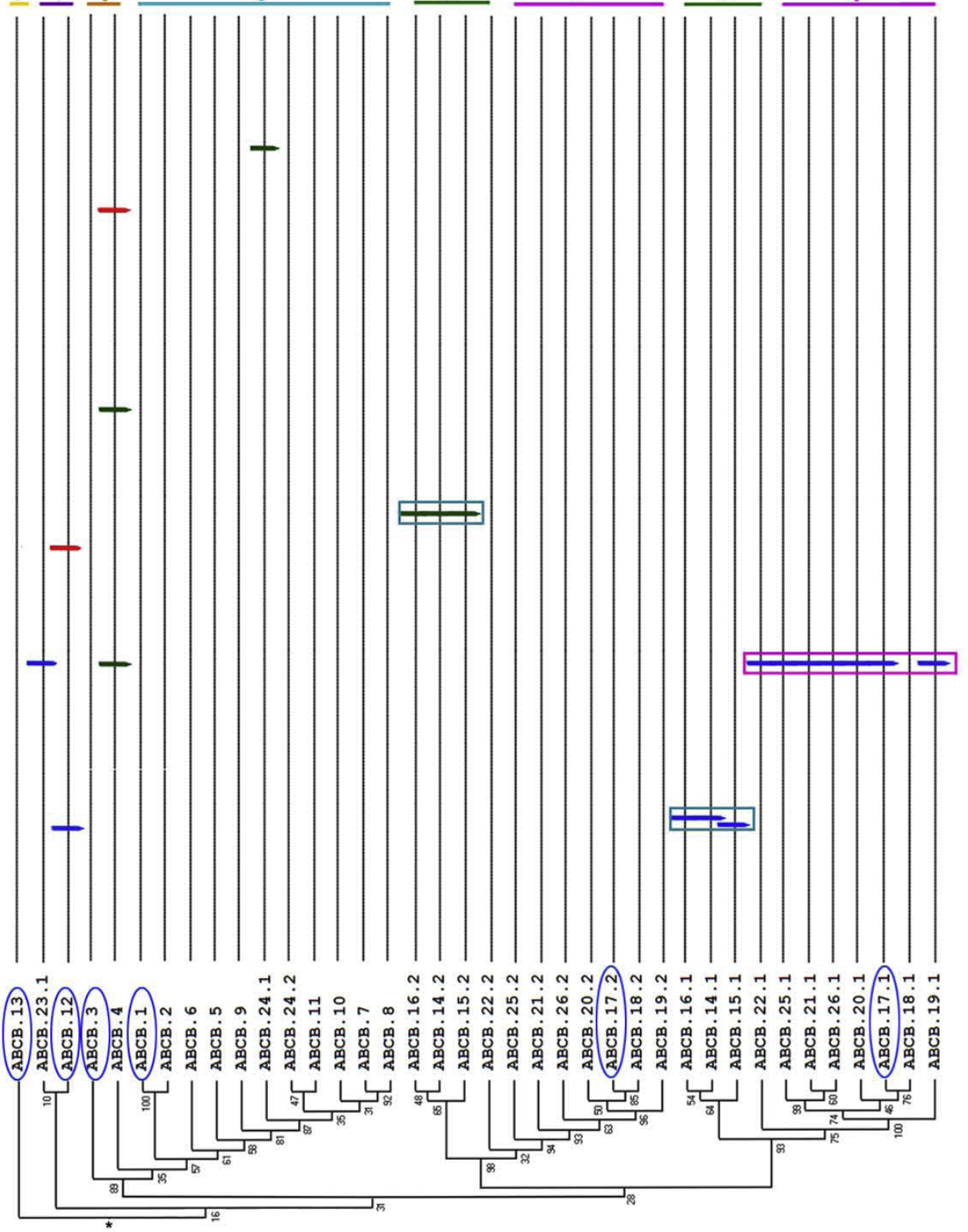

Figure 3 Phylogenetic tree and a proposed evolutionary scheme of $A B C B$ family. Above: the proposed events in the evolution of the $A B C B$ family. a, putative full transporter formation process and divergence of the $A B C B$ transporter with gain and loss of introns, the colored vertical bars in the NBD region represent conserved introns; $b$, divergence of the $A B C B$ transporter gene to give rise to each group. Below: part of tree from Figure 1 with introns locations of NBDs, the asterisk represents the root in the midpoint. Each group was marked as a line with the same color to the pane in b; genes that have a Paramecium ortholog were marked with a blue ellipse. 


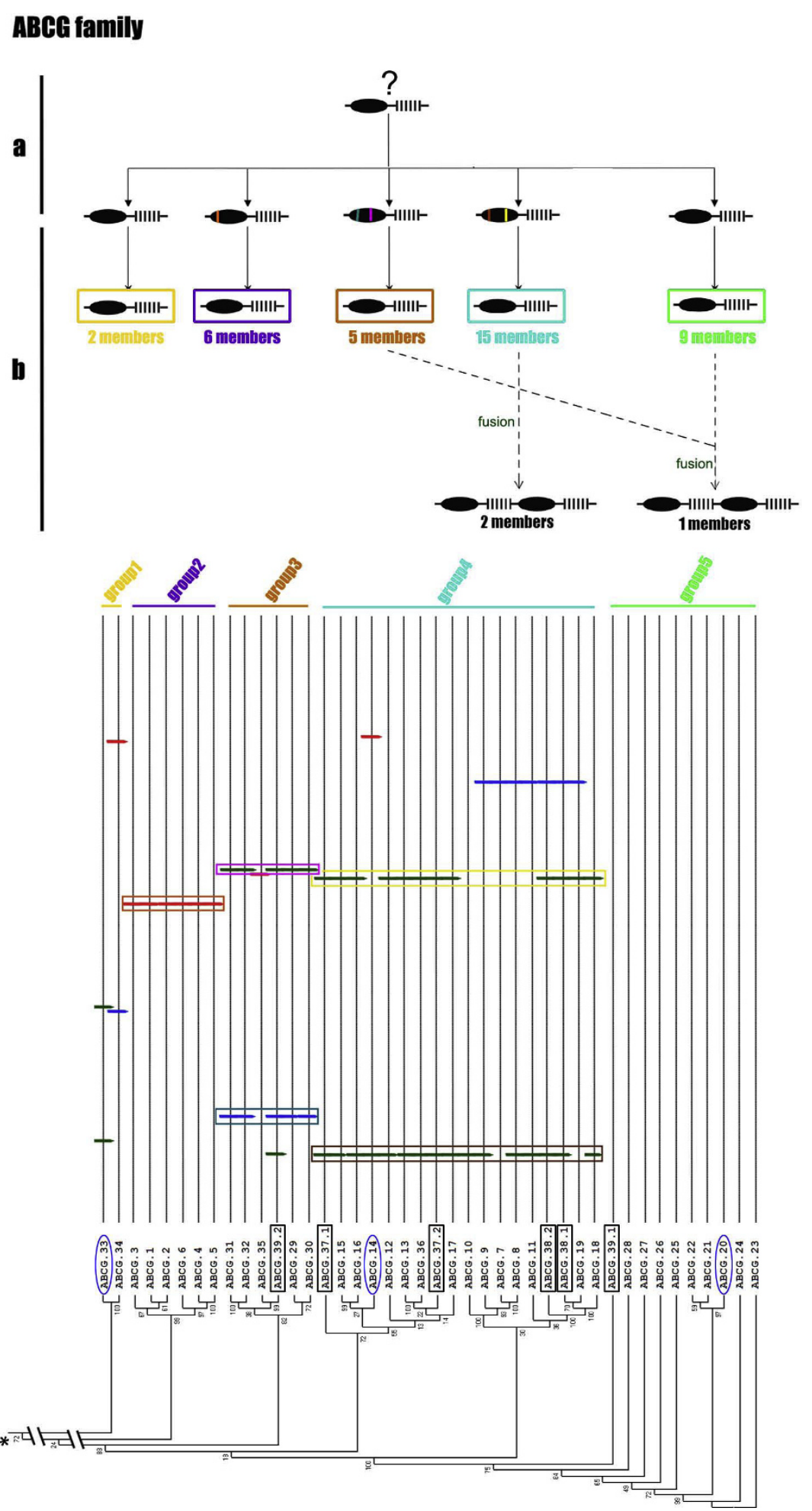

Figure 4 Phylogenetic tree and a proposed evolutionary scheme of ABCG family. Above: the proposed events in the evolution of the ABCG family. a, divergence of the half transporter with intron gain and loss, and the colored vertical bars in the NBD region represent conserved introns; $b$, divergence of the ABCG half transporter gene to give rise to each group, and possible formation process of ABCG full transporters (dash line). Below: part of tree from Figure 1 with introns locations of NBDs, the asterisk represent the root in the midpoint. Each group was marked as a line with the same color to the pane in b. The full transporter NBDs of ABCG are marked with a black pane in the phylogenetic tree; genes that have a Paramecium ortholog were marked with a blue ellipse. 
which may have resulted from recent intron-gain events. Next, full and half transporters in the $A B C A, A B C B$, $A B C C$, and $A B C G$ families were divided into groups. Within each group, the locations of introns were conserved, indicating that intron gain and loss had occurred before the evolutionary expansion of each family. The $\mathrm{ABCA}, \mathrm{ABCB}, \mathrm{ABCC}$, and $\mathrm{ABCG}$ families were massively expanded, containing 17 clusters of tandem repeat genes that comprised a total of 58 genes (Table 2). The ABCG family had the most clusters, six with a total of 29 genes, and its largest single cluster had 11 tandem genes. The sequence identities of proteins in each tandem cluster ranged widely $(50 \%-80 \%)$ and correlated with values of Ks for these tandem genes. However, several tandem repeats had a low Ks (0.1169-0.3109) (Figure 5), and notably, genes within tandem clusters were more closely related to each other than to those in other clusters. This suggests either that duplication events had occurred relatively recently within the clusters of tandem genes or that some mechanism results in the concerted evolution of these genes.

The ABCA family can be divided into 6 groups including two groups of half transporters (groups 5-6 in Figure 2) and four groups of full transporters (groups1-4 in Figure 2), suggesting that Tetrahymena inherited at least $6 \mathrm{ABCA}$ transporter genes from its ancestor. Regarding the expansion of full transporter genes, it was significant that the tree topologies of full transporter NBDs- 1 and NBDs- 2 were surprisingly similar (Figure 2 ). This suggests that these genes evolved as full transporters first and then became expanded. Thus, three genes ancestral to full transporters expanded to form groups1-4, containing 2, 8, 9 and 10 genes, respectively (Figure 2b).

Similar phenomena also were observed in the $\mathrm{ABCB}$ (Figure 3) and $A B C C$ families (data not shown). The ABCG family differed from the others in being composed mainly of expanded half transporters -5 ancestral genes expanded to 2, 6, 5, 15 and 9 genes, respectively (Figure 4b). More than $90 \%$ of the ABCG genes in Tetrahymena are half transporters that are more similar to one another in their sequences than to other $A B C$ transporter families (data not shown), which indicates either

\begin{tabular}{|c|c|c|c|c|c|c|c|}
\hline Family & $\begin{array}{c}2 \\
\text { repeats }\end{array}$ & $\begin{array}{c}3 \\
\text { repeats }\end{array}$ & $\begin{array}{c}4 \\
\text { repeats }\end{array}$ & $\begin{array}{c}5 \\
\text { repeats }\end{array}$ & $\begin{array}{c}6 \\
\text { repeats }\end{array}$ & $\begin{array}{c}11 \\
\text { repeats }\end{array}$ & $\mathrm{n}$ \\
\hline $\mathrm{ABCA}$ & 2 & - & - & - & 1 & - & 3 \\
\hline$A B C B$ & 3 & - & - & - & - & - & 3 \\
\hline $\mathrm{ABCC}$ & 3 & 1 & 1 & - & - & - & 5 \\
\hline ABCG & 2 & 1 & - & 1 & 1 & 1 & 6 \\
\hline
\end{tabular}

very recent expansion, higher negative selection or highly concerted evolution of these genes.

After the phylogenetic analysis of NBDs within Tetrahymena, we compared the detailed compositions of ABC transporter families in Tetrahymena to those of other eukaryotes (Table 1). Tetrahymena, with a predicted proteome of 24725 genes, possesses 53 more ABC transporter genes than those of $P$. tetraurelia, its closest sequenced free-living ciliate which has been shown to have undergone three whole genome duplications (WGDs) [60] resulting in a predicted proteome of 39642 genes. Tetrahymena and Paramecium probably branched off $\sim 800$ Mya, yet only 23 orthologs of ABC transporters between Paramecium and Tetrahymena were found using a reciprocal Blast search (see methods); therefore, $86 \%$ of $\mathrm{ABC}$ genes in Tetrahymena appear to have evolved after divergence of these two species. The pairwise Ks values of 7 sets of $A B C$ transporter genes (see methods) fell mainly between 1.2-2.2 (Figure 5). Rebecca et al (2006) compared the evolutionary rates of proteins among animals, plants and ciliates using $\mathrm{Ka} / \mathrm{Ks}$ and concluded that the genomic architecture of ciliates has experienced the highest rate of evolution, at least 1.6-2.6 times greater than in any other major group of eukaryotes investigated to date [61]. An overall rate of 9.76 mutations per silent site per billion years in Tetrahymena can be calculated using the overall rate of mutations in Arabidopsis (6.1 per silent site per billion years) as a basis for calculation, with a normalization of 1.6 fold, [62]. If this is correct, then most of the duplications of $\mathrm{ABC}$ genes in T. thermophila probably occurred between 61.5-112.7 Mya ago. However, it should be noted that this is a preliminary estimate, and further analysis of the general rate of protein evolution at the genomic level will be required for a more precise calculation.

Comparisons of Tetrahymena with other alvelolate protists, such as the apicomplexans Babesia bovis and Plasmodium yoelii, or more distantly related model organisms, such as S. cerevisiae, D. discoideum, A. thali$a n a$ and $H$. sapiens, also suggest that the superfamily of ABC transporters in Tetrahymena not only has a higher absolute number of genes but also represents a higher percentage of the predicted proteome (Table 1). The large size of this family in Tetrahymena may result from either an increased rate of gene duplication or a low rate of loss. Moreover, it has been shown in Tetrahymena that only genes associated with sensing of and responding to environmental changes (e.g., kinases, voltage-gated ion channels, P-type ATPases and transporter genes) have undergone such large-scale, lineage-specific expansions resulting in a higher number of genes than in other eukaryotic taxa, including the other free-living protists [27]. This suggests that the enlargement of 


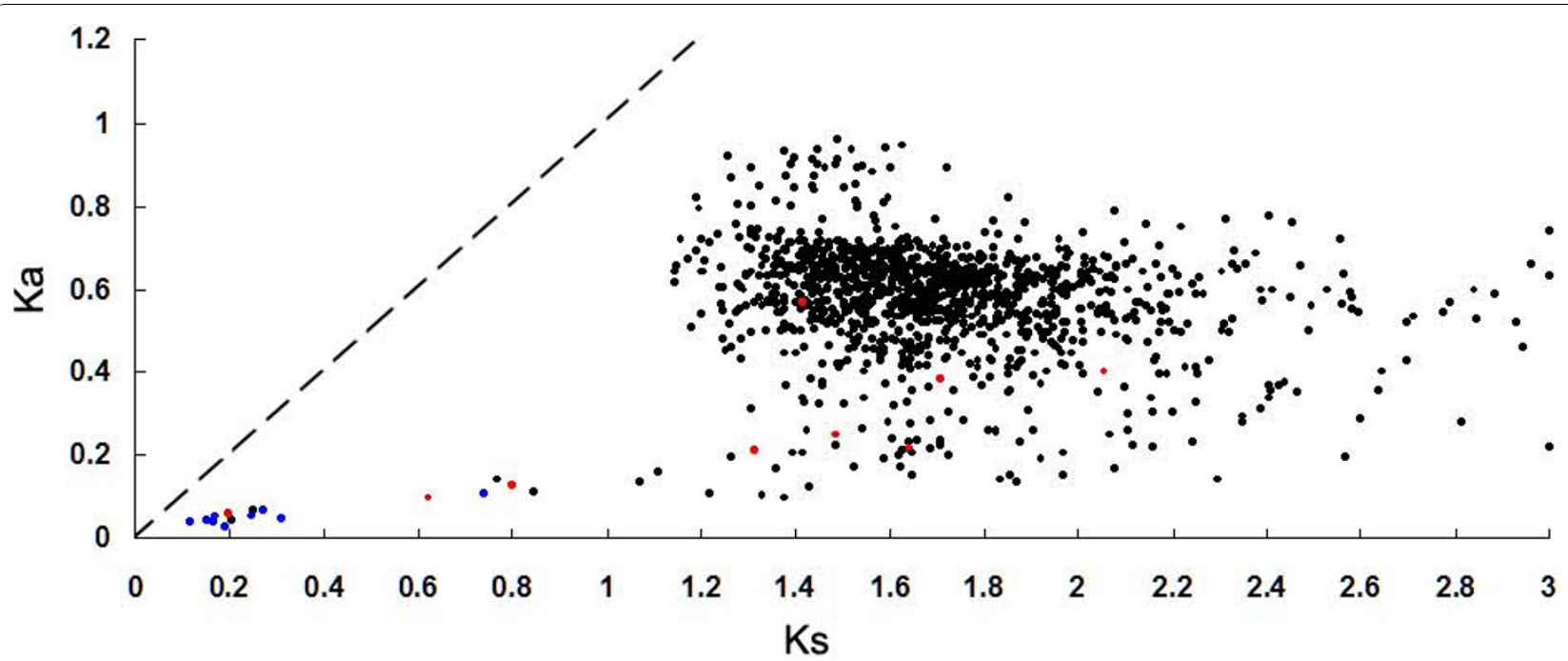

Figure $\mathbf{5}$ Scatter plot of Ka versus Ks. Red dots, pairs of two tandem duplicates; blue dots, pairs of multiple tandem duplicates with a low value of $\mathrm{Ks}$; the dashed line represents $\mathrm{Ka}=\mathrm{Ks}$ (neutral selection); several pairs with $\mathrm{Ks}>3$ were omitted.

several ABC transporter families in T. thermophila resulted from relatively a high duplication rate.

\section{Functional divergence of $A B C$ gene duplicates in Tetrahymena}

Gene duplication is a primary source of new genes with novel or altered functions [63], and a comprehensive classification of all models for the evolution and maintenance of gene duplication has been provided in a recent review [64]. A study of the patterns of expression is one important measure of functions of a gene that could facilitate understanding of the genetic basis of evolutionary change $[65,66]$. The extensive duplications of genes in the $\mathrm{ABC}$ transporter superfamily of Tetrahymena provide a particularly good opportunity to investigate the functional consequences of gene duplication in a single organism.

Figure 6 shows a heat map illustrating the overall expression profiles of $\mathrm{ABC}$ transporters in T. thermophila during different physiological/developmental conditions. Three categories could be identified based on cluster analysis: 1) silent genes or those with low expression levels (some points may silent) (Figure 6a); 2) genes expressed only under specific conditions (Figure 6b);3) genes with high expression levels for all the experimental conditions (Figure 6c). Seven sets of duplicates of $A B C$ genes in the four largest families (ABCA, $A B C B$, $A B C C$ and $A B C G$ ) were selected for analysis of the evolution of their gene-expression patterns. Among these, one set was fit best with the Non-phylogenetic/Free model, and the others all fit best with the Pure-phylogenetic/free model (Table 3). The Pure-phylogenetic model assumes that changes in gene expression occurred on every branch of the phylogeny and that there were neither loss of functions within a gene family in the course of this process nor sporadic changes in gene expression [66]. Thus, either pseudogenization or functional divergence might have occurred in the cases of those six sets of $\mathrm{ABC}$ transporter genes.

We attempted to separate the effects of different modes of duplicate retention by categorizing the expression profile into four types (see methods) for the seven sets of gene duplicates and inferring their ancestral expression states for a comparison. Tandem duplicates of $A B C$ transporters were chosen to represent relatively recent duplications, there being nine such duplicates distributed in six sets of genes (Figure 7). In addition, analyses of those gene sets were performed where applicable, including calculation of $\mathrm{Ka} / \mathrm{Ks}$ values and sites under positive selection, the possibility of forming a heterodimer, and the inducibility to toxic exposure as accessory evidence. The following four different models could be inferred from these nine pairs of duplicates (Table 4).

- Neofunctionalization for four pairs. 1) The ACBA15ABCA16 pair is likely to be undergoing a coding level neofunctionalization, since many sites were detected under positive selection for this pair (Table 4). The loss of expression in one copy could have been shaped by a reduction of the formerly high constitutive expression, to perform a novel function related to, for example, a rare or low-concentration xenobiotics. 2) the ABCA17ABCA18 pair showed the same expression pattern for the ancestral state and two descendants. Similar to the ABCA15-ABCA16, this pair has undergone coding-level neofunctionalization given the relatively high age of the 


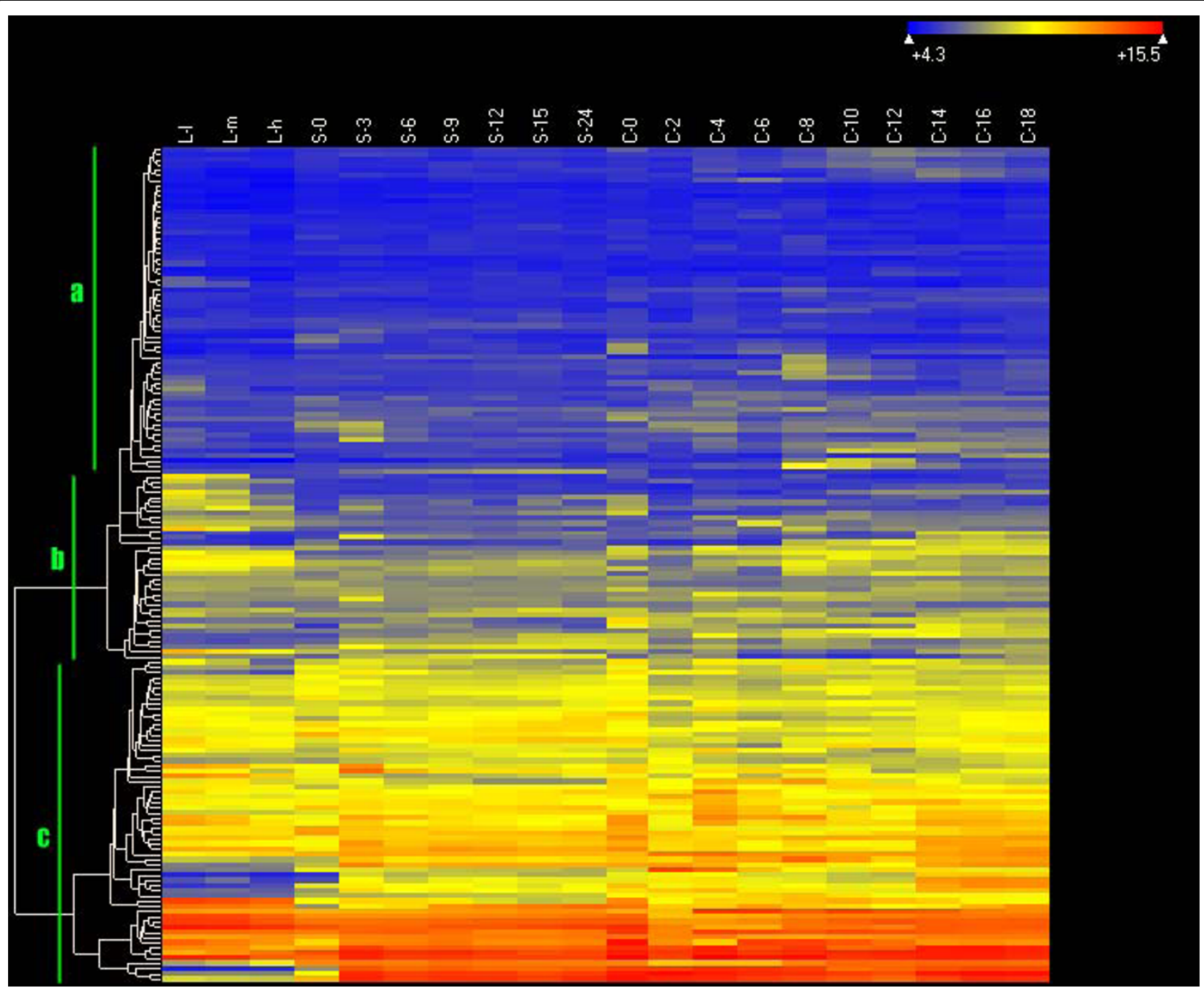

Figure 6 Heat map of the expression of ABC transporter genes in Tetrahymena. The levels of expression are illustrated by different grades of color as determined from microarray data indicated along the top (From left to right). The color scale is as follows: dark color, low expression; light color, high expression. Levels of expression were obtained for 20 points in time during three physiological/development stages of the life cycle of Tetrahymena: For growing cells, L-I, L-m and $\mathbf{L}-\mathbf{h}$ correspond to $\sim 1 \times 10^{5} \mathrm{cell} / \mathrm{s} / \mathrm{ml}, \sim 3.5 \times 10^{5} \mathrm{cell} / \mathrm{s} / \mathrm{ml}$ and $\sim 1 \times 10^{6}$ cells $/ \mathrm{ml}$, respectively. For measurements of expression during starvation, $\sim 2 \times 10^{5}$ cells $/ \mathrm{ml}$ were collected at intervals of $\mathbf{0 , 3}, 6,9,12,15$ and 24 hours (referred to as $\mathbf{S}-\mathbf{0}$, S3, S-6, S-9, S-12, S-15 and S-24, respectively). For measurements of expression during conjugation, equal volumes of B2086 and CU428 cells were mixed together in culture, and samples were collected at intervals of $0,2,4,6,8,10,12,14,16$ and 18 hours after mixing (referred to as $\mathbf{C}$ 0, C-2, C-4, C-6, C-8, C-10, C-12, C-14, C-16 and C-18, respectively). The following three categories could be identified: a, silent genes or those with low expression levels (some points may silent); $\mathrm{b}$, genes highly expressed only for specific conditions; $\mathrm{C}$, genes with continuous high expression levels.

Table 3 The best model of evolution of gene expression for seven sets of genes

\begin{tabular}{ccc}
\hline Sets of genes & \multicolumn{1}{c}{ models } & AIC value \\
\hline ABCA-Set1 & Pure-Phylogenetic/Free & 938.55 \\
ABCA-Set2 & Non-Phylogenetic/Free & 394.963 \\
ABCB-Set1 & Pure-Phylogenetic/Free & 317.005 \\
ABCB-Set2 & Pure-Phylogenetic/Free & 408.603 \\
ABCC-Set1 & Pure-Phylogenetic/Free & 586.047 \\
ABCC-Set2 & Pure-Phylogenetic/Free & 1214.151 \\
ABCG-set1 & Pure-Phylogenetic/Free & 1131.114 \\
\hline
\end{tabular}

duplication and a large number of sites under positive selection (Table 4). 3) the ABCB17-ABCB18 pair had the same type of expression in the ancestral state as the two genes in the pair (Table 4), whereas the ABCB17 gene could be induced by exposure to DDT (unpublished data), suggesting that $\mathrm{ABCB} 17$ might have evolved a new functional role. 4) the ABCC10-ABCC11 pair was inferred as having an ancestral state of high expression for specific conditions still found in the $\mathrm{ABCC} 11$ gene, but $\mathrm{ABCC} 10$ showed a continuous high level of expression (Table 4). Furthermore, positive 


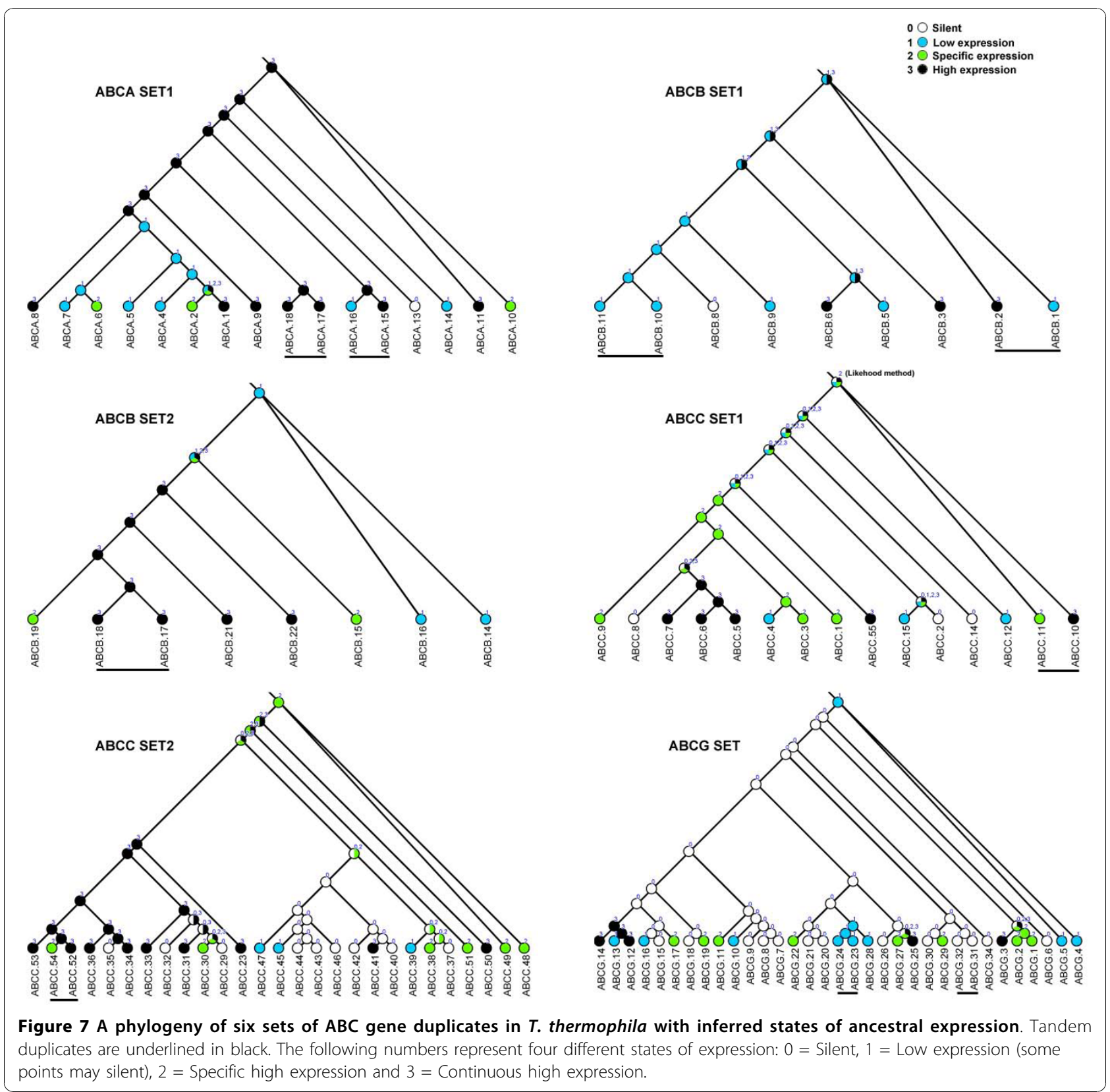

selection on some sites also was detected in the four pairs (Table 4); therefore, the neofunctionalization model was determined to be the best one.

- Subfunctionalization for one pair. The subfunctionalization model implies that duplicates partitioned the function of the ancestral gene and underwent a reciprocal rapid, partial loss of function $[67,68]$. Two expression patterns would be consistent with this model: ancestral ubiquitous expression followed by both duplicates specializing their expression patterns, or high ancestral expression levels with low duplicated pair expression levels (that add up to the previous high level). In our analysis, the ABCB1-ABCB2 showed a relatively recent duplication (with low $\mathrm{Ks}$ ), and more important, the ancestral state may have shown both expression patterns of the descendants prior to duplication (Table 4), therefore, this pair is the most probable duplicates for subfunctionalization. However, simple gradual pseudogenization is another possible explanation for this pair.

- Dosage balance for two pairs. The dosage balance model implies a positive selection for fixation of the duplicates, with both descendants maintaining the original function showing signs of negative selection at the 
Table 4 The best fitted model of the nine tandem duplicates

\begin{tabular}{|c|c|c|c|c|c|c|c|c|c|c|c|c|c|c|c|c|c|c|}
\hline \multirow[t]{2}{*}{ Gene pair } & \multicolumn{4}{|c|}{ ABCA } & \multicolumn{6}{|c|}{ ABCB } & \multicolumn{4}{|c|}{$\mathrm{ABCC}$} & \multicolumn{4}{|c|}{ ABCG } \\
\hline & ABCA15 & ABCA 16 & ABCA17 & ABCA18 & ABCB1 & $\mathrm{ABCB} 2$ & $A B C B 10$ & $\mathrm{ABCB} 11$ & ABCB17 & ABCB18 & $A B C C 10$ & ABCC11 & ABCC52 & ABCC54 & ABCG23 & ABCG24 & ABCG31 & ABCG32 \\
\hline Taxon state & 3 & 1 & 3 & 3 & 1 & 3 & 1 & 1 & 3 & 3 & 3 & 2 & 3 & 2 & 1 & 1 & 0 & 0 \\
\hline Ancestral state & \multicolumn{2}{|c|}{3} & \multicolumn{2}{|c|}{3} & \multicolumn{2}{|c|}{1 or 3} & \multicolumn{2}{|c|}{1} & \multicolumn{2}{|c|}{3} & \multicolumn{2}{|c|}{2} & \multicolumn{2}{|r|}{3} & \multicolumn{4}{|r|}{0} \\
\hline Ks & \multicolumn{2}{|c|}{0.62} & \multicolumn{2}{|c|}{1.71} & \multicolumn{2}{|c|}{0.80} & \multicolumn{2}{|c|}{1.41} & \multicolumn{2}{|c|}{2.05} & \multicolumn{2}{|c|}{1.31} & \multicolumn{2}{|c|}{1.64} & \multicolumn{4}{|c|}{1.49} \\
\hline $\mathrm{Ka} / \mathrm{Ks}$ & \multicolumn{2}{|c|}{0.16} & \multicolumn{2}{|c|}{0.22} & \multicolumn{2}{|c|}{0.16} & \multicolumn{2}{|c|}{0.39} & \multicolumn{2}{|c|}{0.20} & \multicolumn{2}{|c|}{0.16} & \multicolumn{2}{|c|}{0.13} & \multicolumn{4}{|c|}{0.17} \\
\hline Possible heterodimer & \multicolumn{2}{|c|}{ N } & \multicolumn{2}{|c|}{ N } & \multicolumn{2}{|c|}{ N } & \multicolumn{2}{|c|}{ Y } & \multicolumn{2}{|c|}{ N } & \multicolumn{2}{|c|}{ N } & & N & & Y & & N \\
\hline Induced by toxin & N & N & N & N & N & N & N & N & Y (DDT) & N & N & N & N & N & N & N & $N$ & N \\
\hline Positive selection sites & & 1 & 2 & 6 & & 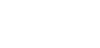 & & 0 & & 2 & & 9 & & 2 & & 4 & & 0 \\
\hline Best model & neofuncti & onalization & neofunctic & onalization & subfuncti & nalization & dosage & balance & neofuncti & onalization & neofuncti & onalization & uncertai & in model & dosage & balance & pseudog & genization \\
\hline
\end{tabular}

Taxon or ancestral expression state: $0=$ Silent, 1 = Low expression (some points may silent), $2=$ Specific high expression and $3=$ Continuous high expression

$\mathrm{Y}=$ yes, $\mathrm{N}=$ no. 
coding level in preservation phase. This model may apply to genes involved in transport [69] and proteinprotein interactions [70]. With low $\mathrm{Ka} / \mathrm{Ks}$ values, two pairs (ABCB10-ABCB11 and ABCG23-ABCG24) showed the same type of expression in the ancestral state as in both duplicates, and these two pairs possibly form heterodimers based on their co-expression PCC values (Table 4), indicating a dosage balance model for these two pairs. The expressed macronuclear genome of Tetrahymena is effectively polyploid (45 copies of each gene on average); however, the retention of both members of a gene pair after duplication to enable an increase in the level of response to match exposure to substances (i.e., increased dosage) may still play a role in the evolution of $\mathrm{ABC}$ transporter genes in T. thermophila.

- Pseudogenization for one pair. The tandem duplicate ABCG31-ABCG32 showed a silent state during the entire life cycle of the T. thermophila (Table 4), and no evidence of transcriptional inducibility from exposure to several toxic substances. The parsimonious ancestral expression state of this pair was also silent (Figure 7). Since the duplication is fairly old and the pairwise Ka/ Ks hints at negative selective pressure operating until quite recently (Table 4), thus, a recent, rapid pseudogenization of both duplicates seems the most probable explanation.

- Uncertain model for one pair. In the ABCC52ABCC54 pair, one descendant (ABCA52) has a high expression level similar to that inferred for the ancestral state, and the other descendant (ABCA54) showed a specific expression pattern. Given the relatively high age of the duplication (high Ks), this pair seems unlikely to be involved in a slow, step-wise subfunctionalization because expression loss of te one copy is expected to be a rapid process. Since only two sites have been detected under positive selection, the possibility that this pair underwent a coding-level neofunctionalization process is also seemingly low. A gradual pseudogenization process that led to the step-wise loss of expression in one copy was possible, where such loss would have to be relatively recent due to the low $\mathrm{Ka} / \mathrm{Ks}$ values of this pair. However, the lack of certainty in regard with their ancestral state further made the situation less clear. Therefore, we thought it is hard to suggest one best model for the ABCC52-ABCC54 pair.

Network asymmetry in which one gene in a pair acquired more co-expressed genes than the other (Additional file 6) could be identified in many tandem duplicate pairs in the seven sets of genes, indicating that each of the two duplicate genes could be involved in a different pathway or biological process. A similar phenomenon also has been reported in yeast [71]. Finally, six genes were found to be up-regulated under stress from DDT and one by exposure to TBT (unpublished data).
This of suggests that there may be more duplicates that have been preserved in the genome through neofunctionalization or dosage balance.

\section{Conclusion}

T. thermophila possesses a total of $165 \mathrm{ABC}$ transporter genes, the largest number in this superfamily reported for any organism to date. The reason for Tetrahymena having so many $\mathrm{ABC}$ transporters is probably related to its lifestyle as a highly dispersed, free-living unicellular organism. For multicellular organisms and parasites, the cells are in more stable environments, whereas Tetrahymena must survive exposure to all the substances and organisms in the fresh water that surrounds it, and thus would benefit from having a large number of diverse genes to enable it to sense and respond to this correspondingly diverse and ever-changing set of environmental conditions. There is evidence that these genes have undergone lineage-specific expansions [27], creating a large, diverse complement of $\mathrm{ABC}$ transporters that could be involved in the efficient efflux of numerous endogenously produced toxins or elimination of a broad range of xenobiotics. The phylogenetic analyses, characterization of intron positions, and ortholog comparisons performed in the present study facilitated both the identification of progenitor genes and formation of strong hypotheses to explain the evolutionary origin and expansion of the $\mathrm{ABC}$ transporter superfamily in Tetrahymena. Our analyses indicated that the fusion of domains or half transporter genes played a role in the evolution of the diversity of $\mathrm{ABC}$ transporters in Tetrahymena along with gene expansion by duplication. These duplicates appear to have experienced subsequent divergence by accumulation of mutations and differences in expression patterns. Multiple different mechanisms including neofunctionalization, subfunctionalization, pseudogenization and dosage balance appear to account for this functional divergence.

\section{Methods}

\section{Identification of genes}

To identify $\mathrm{ABC}$ transporter genes in Tetrahymena, we first searched for predicted ABC transporters genes in the TIGR database (ftp://ftp.tigr.org/pub/data/Eukaryotic_Projects/t_thermophila/Transporter/) and extracted the 160 genes annotated as $\mathrm{ABC}$ transporters. These $A B C$ transporters were divided into full transporters and half transporters, which were aligned separately to identify sequences of NBDs. The NBD sequences then were used to perform a BlastP search (default setting) in TGD [29] to identify other genes with an NBD-like sequence. A total of 185 genes were found, including 1 homolog of Rad50, 4 in the SMC family, 7 in the Muts family, 9 with partial NBD sequences, and 164 with 
complete NBDs. The DNA sequences of these 164 genes were used to do a BlastN search of the NCBI EST database and TBestDB (http://amoebidia.bcm.umontreal.ca/ pepdb/searches/login.php), and the EST information of every gene was examined to determine whether there were any incorrectly predicted genes. RT-PCR was carried out to verify the cDNA sequences, and as a result, one incorrectly predicted gene was separated into two genes, giving a total of 165 predicted $\mathrm{ABC}$ transporter genes with complete NBDs. The protein sequences of these 165 predicted genes were used to perform BlastP searches in the NCBI non-redundant protein database, and hit scores and E-values were sufficient to sort the majority of the $\mathrm{ABC}$ transporter genes into 8 families (several sophisticated genes having to be determined by constructing a phylogenetic tree) [32]. The nomenclature for human $A B C$ families (http://www.genenames. org/genefamily/abc.html) was used to name all $A B C$ transporter genes in Tetrahymena. Orthologs with genes in Paramecium were detected as the reciprocal best hits in the Blast search, using all the $165 \mathrm{~T}$. thermophila $\mathrm{ABC}$ genes as the query.

\section{Sequence alignment, phylogenetic analysis, and intron mapping}

Owing to the difference in length of half and full $A B C$ transporters, we used the NBDs for phylogenetic analysis of $A B C$ transporters. All NBDs were sorted into the following three types: 1 ) NBDs of half transporters were given the same name as the gene (e.g. ABCA29); 2) Nterminal NBDs from full transporters were given their name of their gene followed by .1 (e.g. ABCA1.1); and 3) C-terminal NBDs from full transporters were given the name of their gene followed by .2 (e.g. ABCA1.2). The protein sequences of all NBDs were aligned using MAFFT (http://align.bmr.kyushu-u.ac.jp/mafft/online/ server/index.html) and the matrix Blosum45. Then, the alignment was trimmed using the Trimal program [72] with the option -gt 0.1 , and a maximum likelihood phylogenetic tree was constructed using PhyML [73] with 100 bootstrap iterations.

All nucleotide sequences of NBDs were used to map introns. First, each $\mathrm{ABC}$ transporter $\mathrm{cDNA}$ sequence was aligned with the scaffold sequence to find the position of the introns. Second, the alignment of NBD protein sequences was back-translated into a DNA alignment (codon alignment) of NBDs using RevTrans (http://www.cbs.dtu.dk/services/RevTrans/). Finally, the introns in the NBD regions were mapped manually into the codon alignment of NBD DNA sequences.

\section{Microarray data}

The microarray data were retrieved from the Tetrahymena Gene Expression Database (TGED, http://tged.ihb.ac.cn/).
This included the normalized microarray data published by in Miao et al [28] in which the microarray data of each gene consists of an expression profile containing 20 time points from three stages in the life cycle of $T$. thermophila, including 3 time-dependent points of growth, 7 timedependent points of starvation, and 10 time-dependent points of conjugation. The heat map of the $\mathrm{ABC}$ transporter genes was calculated using the program ArrayStar version 2.0 (DNASTAR, Inc, Madison, WI).

\section{Tetrahymena strains, culture conditions, and toxicity experiments}

The wild-type strain SB210 of T. thermophila was provided by Dr. E. Orias, University of California, Santa Barbara, and strain CU428 was provided by Dr. P.J. Bruns, Cornell University, Ithaca, NY. Cells were grown in SPP medium at $30^{\circ} \mathrm{C}$ [74]. Dr. Y. Xu, Institute of Hydrobiology, Chinese Academy of Sciences, China provided TCDD, which was dissolved in DMSO (Sigma) for use; $\beta$-estradiol was purchased from Sigma. Preparation of DDT was performed as previously described [75]. Aliquots from stock solutions of TCDD, DDT, and $\beta$-estradiol were added to culture medium to produce final concentrations of $1 \mathrm{ppb}, 4 \mathrm{ppm}$, and $1 \mathrm{ppb}$. Cells from stock cultures of CU428 in early stationary phase $\left(\sim 2 \times 10^{5}\right.$ cells $\left./ \mathrm{ml}\right)$ were inoculated into TCDD, DDT, or $\beta$-estradiol-treated media and incubated for 24 hours at $30^{\circ} \mathrm{C}$. The same volume of DMSO used in treatment cultures also was included in cultures used as negative controls.

Total RNA isolation, CDNA synthesis and real-time PCR

Total RNA was isolated using the RNeasy Plus Mini Kit (Qiagen) after cells were homogenized with QIAshredder (Qiagen) according to the manufacturer's instructions. RNA integrity was verified using a Bioanalyzer 2100 (Agilent). RNA samples were treated with DNase (Promega) and subsequently were reverse transcribed into double stranded cDNA using M-MLV reverse transcriptase RNase $\mathrm{H}^{+}$(TOYOBO). Real-time PCR reactions and calculation of the relative expression levels of $A B C B 15$ and $A B C G 25$ were the same as previously described [76].

\section{Analyzing evolution of gene expression and inferring ancestral state of expression}

The procedure used for analyzing evolution of gene expression followed the steps described in [66]. First, the pairwise similarity was calculated using FASTA (http://fasta.bioch.virginia.edu/fasta_www2/fasta_www. cgi). Then, the following two criteria described in [66] were used to partition the superfamily of $A B C$ transporters: 1) sequences must contain a FASTA-alignable region that comprises greater than $80 \%$ of the longer 
protein; 2) two genes resemble each other more than a specified threshold (35\%). Based on these criteria, 7 sets of genes were identified that were suitable for analysis of the evolution of gene expression, including 2 sets each in the $A B C A, A B C B$, and $A B C C$ families and 1 set in the ABCG family. Protein sequences of each set of genes were aligned using MAFFT. Then the alignments were back translated into the codon alignment of DNA sequences using RevTrans.

The GTR+I+G model of evolution was selected by ModelTest [77], and ML trees were constructed by PAUP 4.0b10 [78]. The microarray data of each gene from 20 points in time were $\log 2$-base transformed and represented the character data of taxa tips. Then, both the phylogenetic trees and the log-transformed microarray data were input as a set of continuous data into the program CoMET [79]. Using default settings, 9 maximum likelihood models of the evolution of gene expression were compared according to the Akaike Information Criterion (AIC), and the model with the minimum AIC value was chosen as the best fitting model of evolution of gene expression.

To estimate the best fitting model of the evolution of duplicated $\mathrm{ABC}$ genes, the expression pattern of each of the 7 sets of genes was characterized according to the following system of four types based on the heat map of expression over 20 time points in the life cycle of T. thermophila: 0 , silent; 1 , low expression (some points may silent); 2, specific high expression (in terms of point or stage); 3 , continuous high expression. The ancestral expression states were constructed by parsimony and likelihood methods with the program Mesquite [80] using the four types of expression states $(0,1$, 2 and 3) as characters of taxa.

\section{PCC calculation, searching for candidate co-expressed} genes, and detection of the positive selection

Information on the co-expression of genes of T. thermophila was found in TGED. The Pearson Correlation Coefficient (PCC) of a pair of genes was calculated using a $\mathrm{C}++$-compiled program (available upon request). Candidates for co-expressed genes were identified by the PCC using a threshold of 0.8 (This threshold guaranteed including most genes in KEGG-predicted conserved pathways between T. thermophila and other eukaryotes). Every ABC transporter gene in the 7 sets was searched in TGED, and the candidates for coexpressed genes were obtained. To measure the difference in numbers of candidate co-expressed genes, we adopted the following two methods: 1) calculating the quotient (high number/low number) of candidate coexpressed genes between two genes in a pair and 2) calculating the difference (high number-low number) in candidate co-expressed genes between two genes in a pair. Finally, the percentage of pairs in which the quotient $>2$ and the difference $>100$ was calculated.

The set of 7 genes identified by the procedures given above were used to analyze evolutionary patterns of divergence in expression of genes. Their nucleotide sequences were aligned codon by codon, using the aligned protein sequences as guides, and an ML tree of each set was constructed using PhyML. Then, positive selection was tested by PAML using the branch (Model0), site (Nsites $=1,2,7$ and 8) and branch-site (Model A, specifying the two tandem repeats as foreground lineage) models [81]. In addition, the non-synonymous substitution (Ka) and synonymous substitution (Ks) for gene pairs in each set also were calculated.

\section{Microarray data accession numbers}

Microarray data have been deposited with the NCBI Gene Expression Omnibus (http://www.ncbi.nlm.nih.gov/geo) under accession numbers listed in document S11 in Miao et al [28] ... (doi:10.1371/journal.pone.0004429).

\section{Additional material}

Additional file 1: Characterization of the 165 ABC genes of $T$. thermophila. Families were named according to the nomenclature of Human ABC transporters. Gene ID, structure, intron number, ESTs, protein length and scaffold are listed. a: GST_N_Mu, GST_N family and Class Mu subfamily; ${ }^{\text {b. }}$ Peptidase_S9 domain, Prolyl oligopeptidase family; ${ }^{c}$ : Protein-L-isoaspartate (D-aspartate) O-methyltransferase (PCMT).

Additional file 2: Sketch of ABCB23. The sequence shown here was amplified from CDNA using two primers that are highlighted in red and are matched to regions in NBD-1 and TMD-2. The continuous sequence between NBD-1 and TMD-2 confirmed as ABCB23 was one full transporter and not two half transporters.

Additional file 3: Real-time PCR analysis of expression of $A B C B 15$ and $A B C G 25$ in $T$. thermophila CU428 treated with DMSO, TCDD, DDT, and $\beta$-estradiol. Real-time PCR reactions were performed in triplicate for each CDNA sample, and values were the means of three determinations. DSMO = Dimethyl Sulphoxide; $\mathrm{TCDD}=2,3,7,8$, tetrachlorodibenzo- $p$-dioxin; DDT $=$ dichlorodiphenyltrichloroethane. ${ }^{*} p$ $<0.05$.

Additional file 4: Intron structures of eukaryotic ABCE genes. Each red vertical line represents an intron, $a$ and $b$ represent two ABCE genes in Arabidopsis thaliana. The blue panel represents the intron position conserved between Tetrahymena and Arabidopsis ABCE genes.

Additional file 5: Expression profiles of ABCD1 and ABCD2. 20 time points of the three physiological/development stages of Tetrahymena. For growing cells, $\mathbf{L}-\mathbf{I}, \mathbf{L}-\mathbf{m}$ and $\mathbf{L}-\mathbf{h}$ correspond to $\sim 1 \times 10^{5}$ cells $/ \mathrm{ml}$, $\sim 3.5 \times 10^{5}$ cells $/ \mathrm{ml}$ and $\sim 1 \times 10^{6}$ cells $/ \mathrm{ml}$, respectively. For starvation, $\sim 2 \times 10^{5}$ cells $/ \mathrm{ml}$ were collected at intervals of $0,3,6,9,12,15$ and 24 hours (referred to as S-0, S-3, S-6, S-9, S-12, S-15 and S-24,

respectively). For conjugation, equal volumes of B2086 and CU428 cells were mixed in culture, and samples were collected at intervals of $0,2,4$,

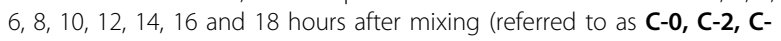
4, C-6, C-8, C-10, C-12, C-14, C-16 and C-18, respectively).

Additional file 6: Different candidates for co-expression of genes between two genes in each pair. The quotient (high number/low number) and the difference (high number/low number) of candidate coexpressed gene number between two genes in each pair have been 
calculated. ${ }^{a}$ : the percentage of pairs in which the quotient $>2{ }^{\text {b: }}$ : the percentage of pairs in which the difference $>100$.

\section{Acknowledgements}

We thank Prof. Martin Gorovsky and Jody Bowen (University of Rochester) for generously sharing the microarray data and for their critical review of the manuscript. We also thank Prof. John Clamp (North Carolina Centra University) for his critical review of the manuscript and comments. This work was supported by grants from the Knowledge Innovation Program of CAS (No. KSCX2-YW-G-072 to MW), National Natural Science Foundation of China (No.30870356), and National Natural Science Foundation of China (No.30970424) to CF. The microarray work was supported by National Institutes of Health grants GM021793 and GM072752 to Prof. Martin Gorovsky when WM was a visiting scientist at the University of Rochester.

\section{Author details}

${ }^{1}$ Key Laboratory of Aquatic Biodiversity and Conservation, Institute of Hydrobiology, Chinese Academy of Sciences, Wuhan, 430072, PR China. ${ }^{2}$ State Key Laboratory of Freshwater Ecology and Biotechnology, Institute of Hydrobiology, Chinese Academy of Sciences, Wuhan, 430072, PR China. ${ }^{3}$ Program in Systematic Biology, Evolutionary Biology Centre, Uppsala 75236, Sweden. ${ }^{4}$ College of Food Science and Biotechnology, Zhejiang Gongshang University, 149 Jiaogong Road, Hangzhou 310035, PR China. ${ }^{5}$ Graduate School of Chinese Academy of Sciences, Beijing, 100049, PR China.

\section{Authors' contributions}

WM, JX and CF conceived and designed the experiments. JX and LF analyzed the data and performed all experiments except the microarray. YD assisted in the experiments. WM performed the microarray experiments. WM, JX, LF and CF interpreted the data and wrote the paper. All authors have read and approved the final manuscript.

Received: 22 February 2010 Accepted: 27 October 2010 Published: 27 October 2010

\section{References}

1. Holland IB, Blight MA: ABC-ATPases, adaptable energy generators fuelling transmembrane movement of a variety of molecules organisms from bacteria to humans. J Mol Biol 1999, 293(2):381-399.

2. Hollenstein K, Dawson RJP, Locher KP: Structure and mechanism of ABC transporter proteins. Curr Opin Struc Biol 2007, 17(4):412-418.

3. Kos V, Ford RC: The ATP-binding cassette family: a structural perspective. Cellular and Molecular Life Sciences 2009, 66(19):3111-3126.

4. Klein I, Sarkadi B, Varadi A: An inventory of the human ABC proteins. Biochim Biophys Acta 1999, 1461(2):237-262.

5. Dean M, Hamon Y, Chimini G: The human ATP-binding cassette (ABC) transporter superfamily. Journal of Lipid Research 2001, 42(7):1007-1017.

6. Gottesman MM, Ambudkar SV: Overview: ABC transporters and human disease. Journal of Bioenergetics and Biomembranes 2001, 33(6):453-458.

7. Gottesman MM, Fojo T, Bates SE: Multidrug resistance in cancer: Role of ATP-dependent transporters. Nature Reviews Cancer 2002, 2(1):48-58.

8. Jones PM, George AM: Multidrug resistance in parasites: $A B C$ transporters, P-glycoproteins and molecular modelling. International Journal for Parasitology 2005, 35(5):555-566.

9. Lanning $\mathrm{CL}$, Fine RL, Corcoran JJ, Ayad HM, Rose RL, AbouDonia MB: Tobacco budworm P-glycoprotein: Biochemical characterization and its involvement in pesticide resistance. Biochimica Et Biophysica Acta-General Subjects 1996, 1291(2):155-162.

10. Aurade R, Jayalakshmi SK, Sreeramulu K: Stimulatory effect of insecticides on partially purified P-glycoprotein ATPase from the resistant pest Helicoverpa armigera. Biochemistry and Cell Biology-Biochimie Et Biologie Cellulaire 2006, 84(6):1045-1050

11. Sreeramulu K, Srinivas R, Jayalakshmi SK: Effect of insecticides on Pglycoprotein ATPase activity of the insecticide resistant pest Helicoverpa armigera. Biochemistry and Cell Biology-Biochimie Et Biologie Cellulaire 2006, 84(6):1059-1060
12. O'Connor R: The pharmacology of cancer resistance. Anticancer Research 2007, 27(3A):1267-1272.

13. Franke RM, Sparreboom A: Drug Transporters: Recent Advances and Therapeutic Applications. Clinical Pharmacology \& Therapeutics 2010, 87(1):3-7.

14. Kroetz $D$ : Involvement of $A B C$ transporters in pharmacokinetics and toxicokinetics. Toxicology Letters 2009, 189:S17-S17.

15. Dean M, Annilo T: Evolution of the ATP-binding cassette (ABC) transporter superfamily in vertebrates. Annual Review of Genomics and Human Genetics 2005, 6:123-142.

16. Roth CW, Holm I, Graille M, Dehoux P, Rzhetsky A, Wincker P, Weissenbach J, Brey PT: Identification of the Anopheles gambiae ATPbinding cassette transporter superfamily genes. Molecules and Cells 2003, 15(2):150-158

17. Sheps JA, Ralph S, Zhao ZY, Baillie DL, Ling V: The ABC transporter gene family of Caenorhabditis elegans has implications for the evolutionary dynamics of multidrug resistance in eukaryotes. Genome Biology 2004, 5(3):R15.

18. Jungwirth $H$, Kuchler $K$ : Yeast $A B C$ transporters - A tale of sex, stress, drugs and aging. Febs Letters 2006, 580(4):1131-1138.

19. Moussatova A, Kandt C, O'Mara ML, Tieleman DP: ATP-binding cassette transporters in Escherichia coli. Biochimica Et Biophysica ActaBiomembranes 2008, 1778(9):1757-1771.

20. Linton KJ, Higgins CF: The Escherichia coli ATP-binding cassette (ABC) proteins. Molecular Microbiology 1998, 28(1):5-13.

21. Davidson AL, Chen J: ATP-binding cassette transporters in bacteria. Annual Review of Biochemistry 2004, 73:241-268.

22. Davidson AL, Dassa E, Orelle C, Chen J: Structure, function, and evolution of bacterial ATP-binding cassette systems. Microbiology and Molecular Biology Reviews 2008, 72(2):317-364

23. Perez-Victoria JM, Parodi-Talice A, Torres C, Gamarro F, Castanys S: ABC transporters in the protozoan parasite Leishmania. Int Microbiol 2001, 4(3):159-166.

24. Klokouzas A, Shahi S, Hladky SB, Barrand MA, van Veen HW: ABC transporters and drug resistance in parasitic protozoa. International Journal of Antimicrobial Agents 2003, 22(3):301-317.

25. Collins K, Gorovsky MA: Tetrahymena thermophila. Current Biology 2005, 15(9):R317-R318

26. Fillingham JS, Chilcoat ND, Turkewitz AP, Orias E, Reith M, Pearlman RE: Analysis of expressed sequence tags (ESTs) in the ciliated protozoan Tetrahymena thermophila. Journal of Eukaryotic Microbiology 2002, 49(2):99-107.

27. Eisen JA, Coyne RS, Wu M, Wu DY, Thiagarajan M, Wortman JR, Badger JH, Ren $\mathrm{QH}$, Amedeo $\mathrm{P}$, Jones KM, et al: Macronuclear genome sequence of the ciliate Tetrahymena thermophila, a model eukaryote. Plos Biology 2006, 4(9):1620-1642.

28. Miao W, Xiong J, Bowen J, Wang W, Liu Y, Braquinets O, Grigull J, Pearlman RE, Orias E, Gorovsky MA: Microarray analyses of gene expression during the Tetrahymena thermophila life cycle. PLOS ONE 2009, 4(2):e4429.

29. Stover NA, Krieger CJ, Binkley G, Dong Q, Fisk DG, Nash R, Sethuraman A, Weng S, Cherry JM: Tetrahymena Genome Database (TGD): a new genomic resource for Tetrahymena thermophila research. Nucleic Acids Research 2006, 34 Database: D500-503.

30. Fu CJ, Xiong J, Miao W: Genome-wide identification and characterization of cytochrome P450 monooxygenase genes in the ciliate Tetrahymena thermophila. Bmc Genomics 2009, 10:208.

31. Sanchez-Fernandez R, Davies TGE, Coleman JOD, Rea PA: The Arabidopsis thaliana $A B C$ protein superfamily, a complete inventory. Journal of Biological Chemistry 2001, 276(32):30231-30244.

32. Anjard C, Loomis WF: Evolutionary analyses of $A B C$ transporters of Dictyostelium discoideum. Eukaryotic Cell 2002, 1(4):643-652.

33. Gbelska Y, Krijger JJ, Breunig KD: Evolution of gene families: the multidrug resistance transporter genes in five related yeast species. Fems Yeast Research 2006, 6(3):345-355.

34. Mengerink KJ, Vacquier VD: An ATP-binding cassette transporter is a major glycoprotein of sea urchin sperm membranes. Journal of Biological Chemistry 2002, 277(43):40729-40734.

35. Iwamoto M, Sugai T, Nakaoka Y: Cell division induced by mechanical stimulation in starved Tetrahymena thermophila: cell cycle without 
synthesis of macronuclear DNA. Cell Biology International 2004, 28(7):503-509.

36. Csere $P$, Lill R, Kispal G: Identification of a human mitochondrial $A B C$ transporter, the functional orthologue of yeast Atm1p. FEBS Letters 1998, 441(2):266-270.

37. Mitsuhashi N, Miki T, Senbongi H, Yokoi N, Yano H, Miyazaki M, Nakajima N, Iwanaga T, Yokoyama Y, Shibata T, et al: MTABC3, a novel mitochondrial ATP-binding cassette protein involved in iron homeostasis. Journal of Biological Chemistry 2000, 275(23):17536-17540.

38. Perez-Tomas R: Multidrug resistance: Retrospect and prospects in anticancer drug treatment. Current Medicinal Chemistry 2006, 13(16):1859-1876.

39. Mcgrath JP, Varshavsky A: The Yeast Ste6 Gene Encodes a Homolog of the Mammalian Multidrug Resistance P-Glycoprotein. Nature 1989, 340(6232):400-404

40. Bamdad M, Reader S, Groliere CA, Bohatier J, Denizeau F: Uptake and efflux of polycyclic aromatic hydrocarbons by Tetrahymena pyriformis: Evidence for a resistance mechanism. Cytometry 1997, 28(2):170-175.

41. Bamdad M, Brousseau P, Denizeau F: Identification of a multidrug resistance-like system in Tetrahymena pyriformis: evidence for a new detoxication mechanism in freshwater ciliates. Febs Letters 1999, 456(3):389-393.

42. Leslie EM, Deeley RG, Cole SPC: Multidrug resistance proteins: role of $\mathbf{P}$ glycoprotein, MRP1, MRP2, and BCRP (ABCG2) in tissue defense. Toxicology and Applied Pharmacology 2005, 204(3):216-237.

43. Depeille P, Cuq P, Mary S, Passagne I, Evrard A, Cupissol D, Vian L: Glutathione S-transferase M1 and multidrug resistance protein 1 Act in synergy to protect melanoma cells from vincristine effects. Molecular Pharmacology 2004, 65(4):897-905.

44. Zolman BK, Silva ID, Bartel B: The Arabidopsis pxa1 mutant is defective in an ATP-binding cassette transporter-like protein required for peroxisomal fatty acid beta-oxidation. Plant Physiology 2001, 127(3):1266-1278.

45. Hettema EH, Distel B, Tabak HF: Import of proteins into peroxisomes. BbaMol Cell Res 1999, 1451(1):17-34.

46. Shani N, Valle D: A Saccharomyces cerevisiae homolog of the human adrenoleukodystrophy transporter is a heterodimer of two half ATPbinding cassette transporters. Proceedings of the National Academy of Sciences of the United States of America 1996, 93(21):11901-11906.

47. Dean M, Rzhetsky A, Allikmets R: The human ATP-binding cassette (ABC) transporter superfamily. Genome Res 2001, 11(7):1156-1166.

48. van Roermund CWT, Visser WF, lijst L, van Cruchten A, Boek M, Kulik W, Waterham HR, Wanders RJA: The human peroxisomal ABC half transporter ALDP functions as a homodimer and accepts acyl-CoA esters. Faseb Journal 2008, 22(12):4201-4208

49. Bisbal C, Martinand C, Silhol M, Lebleu B, Salehzada T: Cloning and Characterization of a Rnase-L Inhibitor - a New Component of the Interferon-Regulated 2-5a Pathway. Journal of Biological Chemistry 1995, 270(22):13308-13317.

50. Marton MJ, deAldana CRV, Qiu HF, Chakraburtty K, Hinnebusch AG: Evidence that GCN1 and GCN20, translational regulators of GCN4, function on elongating ribosomes in activation of elF2 alpha kinase GCN2. Mol Cell Biol 1997, 17(8):4474-4489.

51. Kage K, Tsukahara S, Sugiyama T, Asada S, Ishikawa E, Tsuruo T, Sugimoto Y: Dominant-negative inhibition of breast cancer resistance protein as drug efflux pump through the inhibition of S-S dependent homodimerization. International Journal of Cancer 2002, 97(5):626-630.

52. Xu JK, Liu Y, Yang YY, Bates S, Zhang JT: Characterization of oligomeric human half-ABC transporter ATP-binding cassette G2. Journal of Biological Chemistry 2004, 279(19):19781-19789.

53. Quentin $\mathrm{Y}$, Fichant $\mathrm{G}$ : $\mathrm{ABCdb}$ : an $\mathrm{ABC}$ transporter database. J Mol Microb Biotech 2000, 2(4):501-504.

54. Tomii $\mathrm{K}$, Kanehisa M: A comparative analysis of $A B C$ transporters in complete microbial genomes. Genome Res 1998, 8(10):1048-1059.

55. Saurin W, Hofnung M, Dassa E: Getting in or out: Early segregation between importers and exporters in the evolution of ATP-binding cassette (ABC) transporters. J Mol Evol 1999, 48(1):22-41.

56. Vogel C, Bashton M, Kerrison ND, Chothia C, Teichmann SA: Structure, function and evolution of multidomain proteins. Curr Opin Struc Bio 2004, 14(2):208-216.

57. Garcia O, Bouige P, Forestier C, Dassa E: Inventory and comparative analysis of rice Arabidopsis ATP-binding cassette (ABC) systems. J Mol Biol 2004, 343(1):249-265.
58. Biemans-Oldehinkel E, Doeven MK, Poolman B: ABC transporter architecture and regulatory roles of accessory domains. FEBS Letters 2006, 580(4):1023-1035.

59. Smith PC, Karpowich N, Millen L, Moody JE, Rosen J, Thomas PJ, Hunt JF: ATP binding to the motor domain from an $A B C$ transporter drives formation of a nucleotide sandwich dimer. Mol Cell 2002, 10(1):139-149.

60. Nguyen HD, Yoshihama M, Kenmochi N: The evolution of spliceosomal Introns in alveolates. Molecular Biology and Evolution 2007, 24(5):1093-1096

61. Zufall RA, McGrath CL, Muse SV, Katz LA: Genome architecture drives protein evolution in ciliates. Molecular Biology and Evolution 2006, 23(9):1681-1687.

62. Lynch $\mathrm{M}$, Conery JS: The evolutionary fate and consequences of duplicate genes. Science 2000, 290(5494):1151-1155.

63. Marques AC, Vinckenbosh N, Brawand D, Kaessmann H: Functional diversification of duplicate genes through subcellular adaptation of encoded proteins. Genome Biology 2008, 9(3):R54.

64. Innan H, Kondrashov F: The evolution of gene duplications: classifying and distinguishing between models. Nature Reviews Genetics 2010, 11(2):97-108.

65. Gu X: Statistical framework for phylogenomic analysis of gene family expression profiles. Genetics 2004, 167(1):531-542.

66. Oakley TH, Gu ZL, Abouheif E, Patel NH, Li WH: Comparative methods for the analysis of gene-expression evolution: An example using yeast functional genomic data. Molecular Biology and Evolution 2005, 22(1):40-50.

67. Lynch $M$, Force $A$ : The probability of duplicate gene preservation by subfunctionalization. Genetics 2000, 154(1):459-473.

68. Hughes T, Liberles DA: The pattern of evolution of smaller-scale gene duplicates in mammalian genomes is more consistent with neo-than subfunctionalisation. J Mol Evol 2007, 65(5):574-588.

69. Kondrashov FA, Rogozin IB, Wolf YI, Koonin EV: Selection in the evolution of gene duplications. Genome Biology 2002, 3(2):RESEARCH0008.

70. Veitia RA: Gene dosage balance: deletions, duplications and dominance. Trends Genet 2005, 21(1):33-35.

71. Conant GC, Wolfe KH: Functional partitioning of yeast co-expression networks after genome duplication. Plos Biology 2006, 4(4):545-554

72. Capella-Gutierrez S, Silla-Martinez JM, Gabaldon T: trimAl: a tool for automated alignment trimming in large-scale phylogenetic analyses. Bioinformatics 2009, 25(15):1972-1973.

73. Guindon S, Dufayard JF, Hordijk W, Lefort V, Gascuel O: PhyML: Fast and Accurate Phylogeny Reconstruction by Maximum Likelihood. Infection Genetics and Evolution 2009, 9(3):384-385.

74. Orias E, Hamilton EP, Orias JD: Tetrahymena as a laboratory organism: Useful strains, cell culture, and cell line maintenance. Methods in Cell Biology, Vol 62 2000, 62:189-211.

75. Miao W, Yu T, Orias E, Wan ML, Fu CJ: Identification of differentially expressed genes in Tetrahmena thermophila in response to dichlorodiphenyltrichloroethane (DDT) by suppression subtractive hybridization. Environmental Microbiology 2006, 8(6):1122-1129.

76. Feng LF, Miao W, Wu YX: Difterentially expressed genes of Tetrahymena thermophila in response to tributyltin (TBT) identified by suppression subtractive hybridization and real time quantitative PCR. Aquatic Toxicology 2007, 81(1):99-105

77. Posada D, Crandall KA: MODELTEST: testing the model of DNA substitution. Bioinformatics 1998, 14(9):817-818.

78. Swofford DL: PAUP*: Phylogenetic Analysis Using Parsimony (and Other Methods) 4.0 Beta. Sinauer Associates, Sunderland, Mass; 2001.

79. Lee C, Blay S, Mooers AO, Singh A, Oakley TH: CoMET: A Mesquite package for comparing models of continuous character evolution on phylogenies. Evol Bioinform Online 2006, 2:183-186.

80. WP Maddison DM: Mesquite: a modular system for evolutionary analysis. Version 2.5. 2008 [http://mesquiteproject.org].

81. Yang ZH: PAML: a program package for phylogenetic analysis by maximum likelihood. Computer Applications in the Biosciences 1997, 13(5):555-556.

doi:10.1186/1471-2148-10-330

Cite this article as: Xiong et al: Genome-wide identification and evolution of ATP-binding cassette transporters in the ciliate Tetrahymena thermophila: A case of functional divergence in a multigene family. BMC Evolutionary Biology 2010 10:330. 